\title{
Enfermedad de Chagas del sistema nervioso central en un paciente con SIDA demostrada por métodos cuantitativos moleculares
}

\author{
Alberto Fica, Marcia Salinas, María Isabel Jercic, Jeannette Dabanch, \\ Andrés Soto, Sergio Quintanilla y Carlos Flores
}

\section{Chagas disease affecting the central nervous system in a patient with AIDS demonstrated by quantitative molecular methods}

Although infrequent, Trypanosoma cruzi reactivation is possible among patients with HIV/AIDS infection that develop a tumor-like or granulomatous lesion in the CNS. We report the case of a 60 years old male patient with HIV /AIDS and low CD4 lymphocytes count with cerebellar symptoms and mild paresis, associated to supra and infratentorial hypodense lesions and positive serology tests both to T. gondii and Trypanosoma cruzi. Empirical therapy against toxoplasmosis was prescribed together with antiretroviral therapy but without a favorable response. Brain Chagas disease was confirmed by quantitative PCR in the CSF but he died despite nifurtimox treatment. Despite its rare occurrence, Chagas disease affecting the CNS is possible among patients with HIV/AIDS infection. Epidemiological exposure, a positive Chagas serological test and the image pattern of brain lesions support the suspicion. Diagnosis can be confirmed by molecular test in CSF samples, including new quantitative methods. Despite an adverse prognosis, specific therapy can be attempted besides antiretroviral treatment.

Key words: Chagas disease; central nervous system; acquired immunodeficiency syndrome; real-time polymerase chain reaction; parasite load; nifurtimox.

Palabras clave: Enfermedad de Chagas; sistema nervioso central; SIDA; RCP en tiempo real; carga parasitaria; nifurtimox.

\section{Introducción}

$$
\mathrm{L}
$$

a infección por Trypanosoma cruzi se denomina enfermedad de Chagas. Representa un importante problema de salud pública mundial e integra la lista de infecciones desatendidas. Se estima que en el mundo existen cerca de 7 millones de personas infectadas y que anualmente fallecen unas 10.000 personas por las complicaciones asociadas ${ }^{1}$. La infección es producida por un parásito flagelado que es transmitido por un insecto triatomino hematófago. Evoluciona como un cuadro agudo (fase aguda), o bien con formas crónicas asintomáticas (fase crónica indeterminada) o sintomáticas (fase crónica determinada) por la afección de órganos blanco como corazón, esófago y colon. También pueden ocurrir reactivaciones de infecciones latentes en hospederos inmunocomprometidos y que pueden llegar a comprometer el sistema nervioso central (SNC) y/o el miocardio.

Además de la infección vectorial, puede transmitirse en forma vertical durante el embarazo, por transfusión sanguínea en ausencia de tamizaje, por órganos trasplantados o por ingestión de alimentos contaminados ${ }^{2-6}$. Esta infección ocurre sólo en el continente americano pero ha sido observada en otros continentes por migraciones humanas, trasplantes de órganos o donaciones de sangre ${ }^{5,7}$. En su fase aguda, la enfermedad de Chagas tiene tratamiento y en la fase crónica, donde se afectan el corazón (hasta en $30 \%$ de los casos) y el tubo digestivo (hasta $10 \%$ ), este tratamiento también puede moldear su evolución.

En Chile, en el año 2000 se declaró la interrupción de la transmisión vectorial de la enfermedad de Chagas pero el vector sigue estando presente ${ }^{8}$ y continúa operativa la transmisión vertical la que se acopla a una enorme cohorte de pacientes en la fase crónica indeterminada.

La enfermedad de Chagas en pacientes inmunocomprometidos ha sido descrita especialmente en asociación al trasplante de órganos sólidos y en pacientes con coinfección VIH/SIDA, siendo en estos casos de evolución más grave y letal. La coinfección de VIH-Chagas es muy infrecuente y ello atenta contra la adecuada sospecha y manejo de estos pacientes. El propósito de esta comunicación es presentar un caso de infección del SNC por tripanosomiasis americana en un paciente con infección por VIH en etapa SIDA y analizar los problemas de diagnóstico diferencial, confirmación de laboratorio, manejo y pronóstico.
Hospital Militar de Santiago. Servicio de Infectología (AF, JD, AS) Servicio de Imagenología (MS). Servicio de Neurología (SQ, CF). Instituto de Salud Pública de Chile. Sección Parasitología (MIJ).

Recibido: 19 de julio de 2016 Aceptado: 12 de enero de 2017

Correspondencia a: Alberto Fica Cubillos albertoficacubillos@gmail.com 


\section{Caso clínico}

Paciente de sexo masculino de 60 años, con antecedentes de haber residido en forma transitoria en Ovalle, Región de Coquimbo, por motivos laborales hacía aproximadamente 20 años, además de una parálisis facial periférica un año antes. Consultó por un cuadro de pérdida de peso de $8 \mathrm{~kg}$ en dos meses, vómitos repetidos y un posterior trastorno del equilibrio y vértigo. Al ingreso, se encontraba enflaquecido, consciente y orientado, sin otros hallazgos al examen general. El examen neurológico se constató sin afasia, con prueba positiva de mínima paresia, dismetría y disdiadococinesia izquierdas. Entre los exámenes de laboratorio tenía un hemograma, proteína $\mathrm{C}$ reactiva, función renal, perfil hepático, coagulación y orina completa normales, salvo una albuminemia de 3,4 g/ dl. La tomografía computarizada (TC) cerebral reveló un edema vasogénico con una lesión tipo masa en el lóbulo frontal derecho; y otra imagen parietal con impregnación de contornos irregulares al contraste. La resonancia magnética (RM) cerebral confirmó la presencia de al menos tres lesiones hipointensas con impregnación marginal con medio de contraste y significativo edema perilesional de ubicación frontal, con características de granulomas, y otras similares a nivel frontoparietal derecho y en fosa posterior a nivel cerebelar izquierdo, menores a $2,6 \mathrm{~cm}$, sugerentes de una probable toxoplasmosis cerebral (Figura $1 \mathrm{~A}, 2 \mathrm{~A}$ y $3 \mathrm{~A})$. El examen físico segmentario reveló una candidiasis orofaríngea y leucoplaquia vellosa y por la sospecha de una toxoplasmosis cerebral se inició terapia con cotrimoxazol en dosis $8-10 \mathrm{mg} / \mathrm{kg} /$ día (en base a trimetoprim), corticoesteroides sistémicos (dexametasona $12-16 \mathrm{mg} /$ día) y fluconazol oral (150 mg/día v.o. por 10 días). Las serologías por quimioluminiscencia de VIH y ELISA de $T$. cruzi resultaron reactivas, siendo ambas confirmadas posteriormente en el laboratorio de referencia nacional (ELISA e IFI positivas para Chagas) y se confirmó además serología positiva IgG para Toxoplasma gondii. Las serologías para sífilis, hepatitis $\mathrm{B}$ y $\mathrm{C}$ fueron negativas, sin detectar anomalías al fondo de ojo. El análisis de LCR no demostró alteraciones en sus valores y el estudio para sífilis, bacterias, hongos y tuberculosis fue negativo. No se hizo estudio molecular en esta primera punción lumbar. Posteriormente, se obtuvo recuento de linfocitos CD4 de 73 cél $/ \mathrm{mm}^{3}$ y una carga viral VIH de 54.438 copias $/ \mathrm{ml}(\log 4,74)$. El estudio por TC de tórax, abdomen y pelvis no mostró adenopatías, visceromegalias ni lesiones residuales de tuberculosis.

El paciente evolucionó con rechazo alimentario, ánimo bajo y labilidad emocional. Se diagnosticó un trastorno depresivo/adaptativo grave, que se trató con mirtazapina y olanzapina. A los 10 días de tratamiento con cotrimoxazol y 26 de hospitalización se inicio terapia antiretroviral (TARV) con abacavir, lamivudina y raltegravir, que toleró en forma adecuada y con estudio previo genotípico para HLA*B5701 negativo. Se desestimó el uso de inhibidores de proteasa por eventuales interacciones con psicofármacos y de efavirenz por la depresión concurrente. Durante

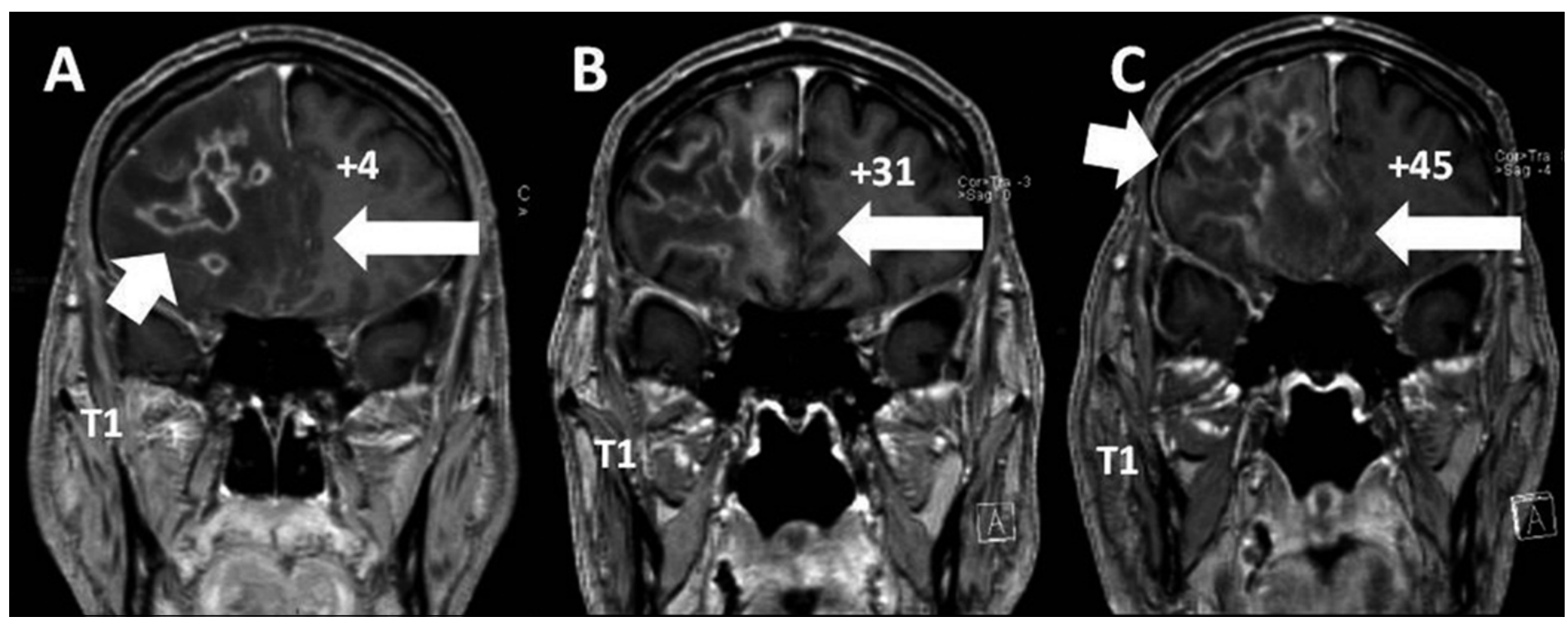

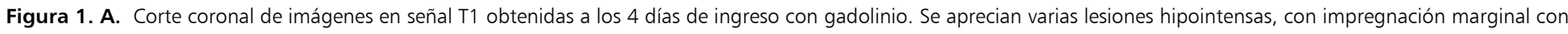

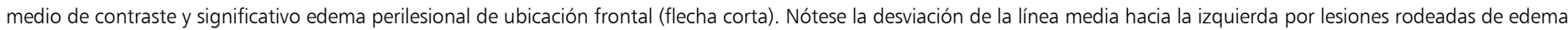

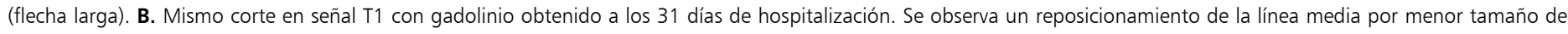

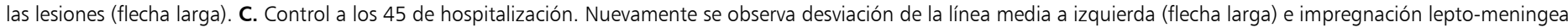
frontal derecha (flecha corta). 
los primeros días del inicio de la TARV el paciente presentó diplopía, nistagmus y estrabismo convergente del ojo derecho, asociado a una marcada agresividad. Se controló una nueva RM cerebral a los 15 días de terapia con cotrimoxazol (+31 días de hospitalización), que mostró una disminución significativa de las lesiones frontales y del edema (Figura 1B y 2B) pero con lesiones cerebelares estacionarias con mayor edema perilesional. Se planteó la realización de un estudio histológico de las lesiones, para descartar un linfoma primario de SNC y otros diagnósticos diferenciales. No obstante, debido a que dichas lesiones radiológicamente no empeoraron, el equipo de Neurocirugía desestimó realizar una biopsia abierta.

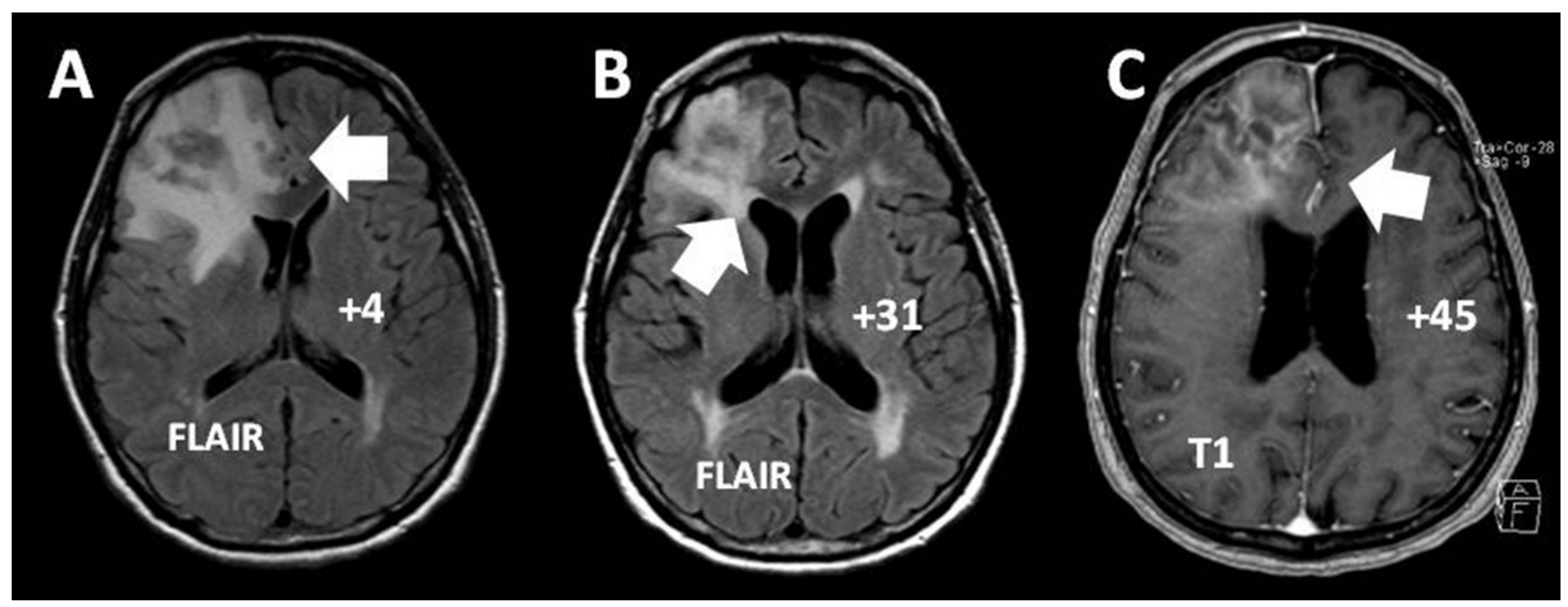

Figura 2. A. Imagen FLAIR (Fluid Attenuated Inversion Recovery) en corte transverso obtenida a los 4 días de ingreso. Se aprecia hiperintensidad por edema, que rodea las lesiones y que determinan un efecto de masa ya que desplaza la línea media (flecha). La técnica FLAIR permite una mejor visualización del edema sin sobreposición con la señal que da el LCR. En contraste, la señal T2 mostraría igual hiperintensidad para el LCR y el edema impidiendo ver sus límites. B. Control a los 31 días de hospitalización con la misma técnica que revela una disminución del edema cerebral y reposicionamiento de los cuernos ventriculares frontales (flecha). C. Imagen en señal T1 en corte transverso con gadolinio a los 45 días desde el ingreso. Se aprecia aumento volumétrico de la lesión frontal izquierda que desplaza las arterias cerebrales anteriores a izquierda de la línea media (flecha).

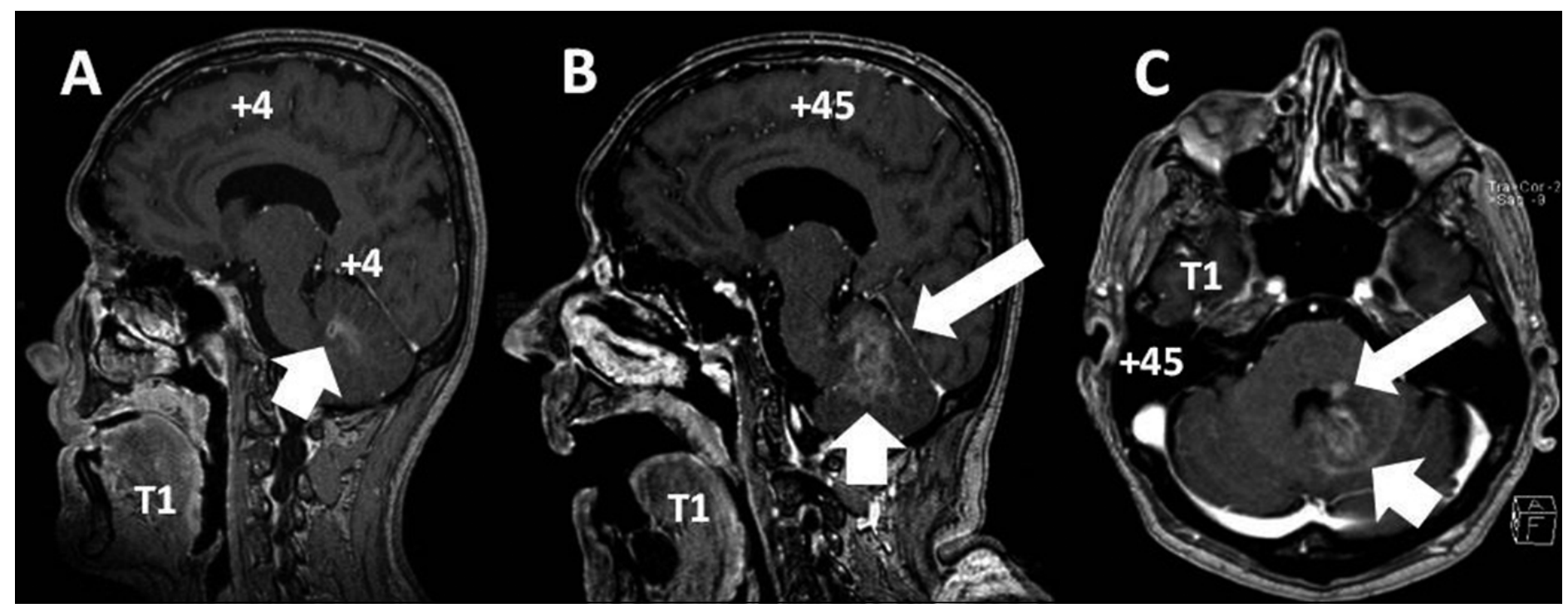

Figura 3. A. Imagen en señal T1 en corte sagital con gadolinio a los 4 días de ingreso. Se aprecia una lesión cerebelosa con impregnación anular (Flecha). B. Control a los 45 días en señal T1 con gadolinio que revela la progresión de la lesión cerebelosa con crecimiento hacia craneal (flecha corta) y aproximación al tentorio (flecha larga). C. Control a los 45 días en señal T1 en corte transverso con gadolinio. Se aprecia aumento volumétrico de lesión cerebelosa izquierda que comprime lateralmente el cuarto ventrículo (flecha corta). Se nota también, la aparición de nueva lesión nodular protuberancial posterior izquierda (flecha larga). 
Durante la cuarta semana de terapia con cotrimoxazol y tercera de TARV, inició fiebre hasta $38^{\circ} \mathrm{C}$, somnolencia hasta el sopor profundo, paresia de hemicuerpo izquierdo, del velo palatino izquierdo y disartria intensa, objetivándose una infección del tracto urinario por Klebsiella pneumoniae multiresistente. Un nuevo control de RM cerebral, al mes de tratamiento con cotrimoxazol, confirmó la progresión de las lesiones cerebrales, especialmente de la fosa posterior izquierda con mayor edema de la lesión frontal derecha y aparición de nuevas lesiones supra e infratentoriales, efecto de masa y herniación subfalcina a izquierda (Figura 1C, 2C, 3B y 3C).

Se planteó un probable compromiso de SNC por enfermedad de Chagas o un síndrome inflamatorio de reconstitución inmune (SIRI), por lo que se decidió trasladar a UCI ese mismo día para protección de la vía aérea (escala Glasgow de coma 5-6 puntos) con ventilación mecánica invasora y monitorización, manteniéndose la TARV, cotrimoxazol y corticoesteroides. Se inició empíricamente nifurtimox $8 \mathrm{mg} / \mathrm{kg} /$ día $(120$ mg fraccionado cada 6 horas por SNG), asociado a meropenem i.v. para el manejo de la infección urinaria. Se amplió el estudio etiológico con una carga viral para citomegalovirus en sangre que fue indetectable y

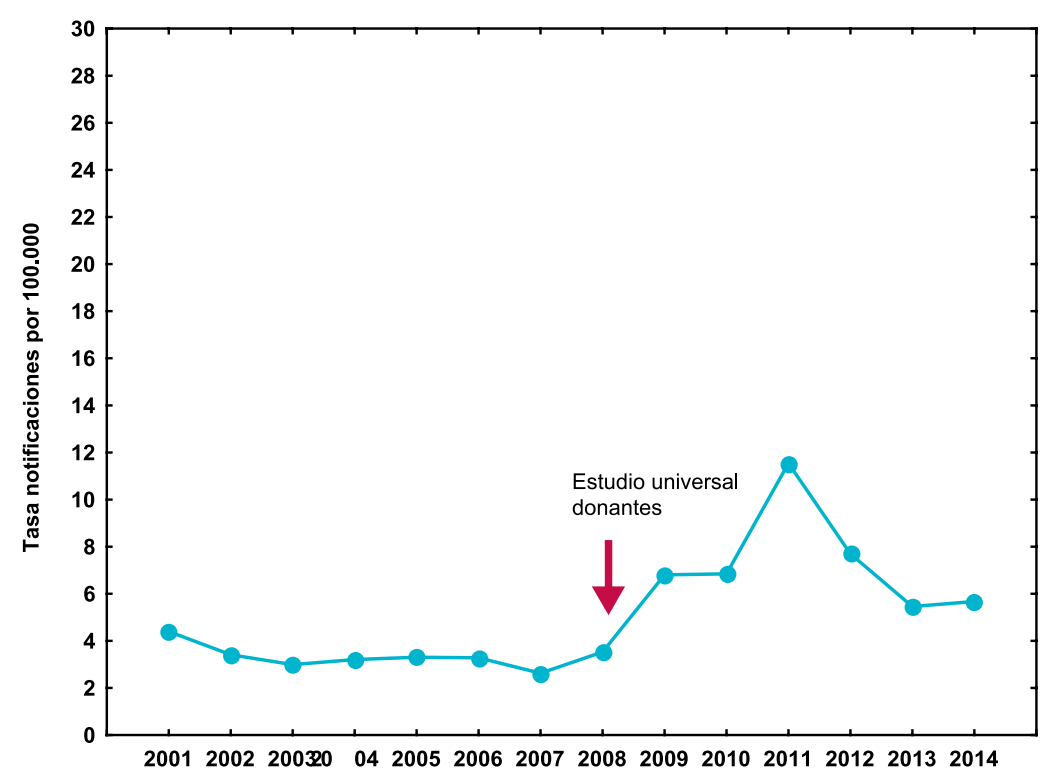

Figura 4. Tasa notificación poblacional de Enfermedad de Chagas, Chile 2001-2015. Gráfico construido con datos del archivo Serie histórica ENO 1950-2014 WEB y población nacional estimada para Chile según datos del INE/ CEPAL, disponible en páginas www.deis.cl y http://www.ine.cl/canales/chile_estadistico/demografia_y_vitales/proyecciones/Informes/MicrosoftWordInforP_T.pdf. Sitios visitados en junio del 2016. La flecha indica el momento en que se amplía el tamizaje para T. cruzi en donantes de sangre en zonas endémicas a todo el país y que pudo haber influido en el aumento de las tasas estimadas. hemocultivos para micobacterias que resultó negativo. Se realizó una segunda punción lumbar, con cultivo corriente, de hongos y tinta china en LCR nuevamente negativos, pero apareció una proteinorraquia de $55 \mathrm{mg} /$ dL. El estudio complementario del LCR con tinción de tripomastigotes, RPC con Xpert MTB/RIF para Mycobacterium tuberculosis y RPC de virus herpes simplex-1 y 2, varicela zoster, Epstein-Barr y JC también fueron negativos. Además, la RPC para $T$. gondii en sangre periférica y LCR también fue negativa teniendo como target el gen multicopia B1 ${ }^{9}$. Sin embargo, la RPC para T. cruzi en sangre reveló 1.400 copias $/ \mathrm{mL}$, y 35.000 copias/mL en el LCR, confirmándose una encefalitis chagásica. Los targets utilizados para la detección de $T$. cruzi fueron para el minicírculo contenido en el ADN de kinetoplasto $(\mathrm{ADNk})^{10}$ y la región repetitiva de 195 pb contenida en el núcleo, denominado ADN satélite (ADNsat). ${ }^{11}$ En su quinta semana de TARV se realizó control de carga viral de VIH que fue indetectable (con reducción $>1 \log$ respecto a la basal), sin aumento en el recuento CD4 (30 céls $/ \mu \mathrm{L}$ ), aunque el paciente estaba recibiendo altas dosis de corticoesteroides.

El paciente evolucionó con extrema gravedad, necesidad continua de VM y aminas vasoactivas, sin mayor respuesta neurológica a terapias instauradas. Luego de dos meses de hospitalización falleció por un síndrome de hipertensión intracraneal y falla multiorgánica.

\section{Discusión}

Varios factores parecen explicar la baja frecuencia de co-infección de VIH/SIDA con la enfermedad de Chagas. Estos incluyen la baja frecuencia relativa de ambas condiciones (aproximadamente 10 casos nuevos por 100.000 habitantes cada año para VIH/SIDA y cerca de 6 por 100.000 para enfermedad de Chagas) (Figura 4) ${ }^{12}$, la mayor concentración urbana de los casos de VIH/SIDA (contrastando con el origen más bien rural de la infección por $T$. cruzi ), el tamizaje de hemoderivados para este parásito (una estrategia universal aplicada desde el 2008 en todo Chile pero de mayor antigüedad en las zonas endémicas de Chagas), la concentración de casos de VIH/SIDA (centro-sur del país) en zonas no endémicas para enfermedad de Chagas (norte de Chile) y finalmente la interrupción de la transmisión vectorial documentada desde hace más de una década en nuestro país. Estos factores han limitado la aparición de la infección chagásica en pacientes con infección VIH/SIDA en nuestro país y explican su aparición esporádica y anecdótica, la que en ocasiones ha estado ligada a la recepción de hemoderivados en otros países $^{13-16}$. Estimaciones efectuadas en Brasil indican una tasa de co-infección de aproximadamente $1,3 \%$ y cerca 
de 21.000 casos coinfectados para Latinoamérica ${ }^{17}$. Esta baja frecuencia atenta contra su sospecha clínica, diagnóstico y terapia oportuna. Además, este tímido perfil epidemiológico hace que la sospecha sea derivada hacia la toxoplasmosis cerebral, la infección oportunista más frecuente del SNC en nuestro medio en pacientes con VIH/SIDA, tal como ocurrió en nuestro caso. De hecho, en un estudio retrospectivo de nueve años (2005-2013) en pacientes con VIH/SIDA que ingresaron a unidades críticas o semicríticas en nuestro hospital, no se detectó ningún caso de infección chagásica pero sí dos casos de toxoplasmosis cerebral en el mismo período, entre 32 pacientes analizados ${ }^{18}$. No fue sino hasta que se hubiera considerado el fracaso de la terapia con corticoesteroides y cotrimoxazol, iniciados empíricamente por la sospecha de toxoplasmosis, que se planteó la posibilidad de una infección cerebral oportunista por $T$. cruzi. Según nuestros antecedentes, no se ha descrito un infección cerebral simultánea por T. cruzi y $T$. gondii en pacientes con VIH/SIDA por lo que la opción de haber tratado las dos condiciones simultáneamente no tenía base científica y además exponía al paciente a reacciones adversas o interacciones farmacológicas. El diagnóstico diferencial entre ambas condiciones es difícil en ausencia de un estudio invasor. Estudios de series de casos estudiados con autopsias indican que la enfermedad de Chagas cerebral tiene compromiso de la materia gris y blanca, pero predominantemente en ésta última, se localizan en varios lóbulos cerebrales donde alcanzan su mayor tamaño y también se ubican en el cerebelo y tronco cerebral, pero sin compromiso de los ganglios basales. En contraste, los pacientes con toxoplasmosis cerebral, tiene compromiso de corteza cerebral o sustancia blanca pero a nivel subcortical y casi todos presentan compromiso de los ganglios basales ${ }^{19,20}$. Sin embargo, en ocasiones los pacientes con toxoplasmosis cerebral pueden presentar lesiones infratentoriales ${ }^{20}$. La evolución de las imágenes en nuestro paciente fue confirmando este patrón con compromiso supra e infratentorial, cerebeloso y de tronco sin afectar los ganglios basales.

El sitio anatómico más afectado por la enfermedad de Chagas en pacientes con VIH/SIDA corresponde al SNC (cerca de $75 \%$ de los casos), siendo secundado por compromiso miopericárdico o en ambos sitios ${ }^{15,17,21,22}$. Habitualmente, la infección ocurre por reactivación de una infección latente adquirida previamente ${ }^{15,17}$. En pacientes con VIH/SIDA, el promedio descrito de recuento de linfocitos CD4 para casos de reactivación es de 98 céls/ $\mu \mathrm{L}$ (rango 1-551) y de 561 céls $/ \mu \mathrm{L}$ para casos no asociados a reactivación (rango 44-1.949). La sobrevida es menor en los casos reactivados en comparación a los no asociados a reactivación (10,6 meses versus 2,8 años; respectivamente) ${ }^{17}$. Así, el compromiso neurológico presentado por el paciente, la probable reactivación de una infección latente adquirida por viajes remotos a una zona endémica, el bajo recuento de linfocitos $\mathrm{CD} 4$ al momento del diagnóstico y su curso letal en corto tiempo, están en concordancia con lo observado en la literatura especializada ${ }^{15,17}$. Los factores asociados a un peor pronóstico incluyen el compromiso del SNC, el diagnóstico tardío de la infección chagásica y el inicio tardío de la terapia específica, todos ellos presentes en nuestro caso ${ }^{17}$.

Las manifestaciones clínicas del compromiso del SNC en pacientes con infección VIH-Chagas son inespecíficas y corresponden a cefalea, signos de hipertensión endocraneana, convulsiones, focalización o coma. Este perfil puede simular un tumor del $\mathrm{SNC}^{13,15,16,23,24}$. El riesgo de reactivación en pacientes con infección chagásica y VIH es de 15 a $21 \%$ en estudios de seguimiento y está asociado al nivel de inmunosupresión ${ }^{25}$.

Se han descrito algunos pocos casos con serología positiva para $T$. gondii y $T$. cruzi en forma simultánea que han sido tratados inicialmente como toxoplasmosis, sin respuesta como en nuestro caso, pero con demostración de una infección monomicrobiana chagásica luego de realizar un estudio invasor ${ }^{26}$.

El diagnóstico del compromiso chagásico del SNC se establece por la detección directa de tripomastigotes flagelados en el LCR al examen al fresco o por tinción Giemsa, por la presencia de amastigotes en el tejido de biopsia o autopsia o cultivo en medios especiales ${ }^{14,22,26-28}$. En años más recientes se ha desarrollado el diagnóstico cuantitativo por métodos moleculares como el aplicado en nuestro paciente ${ }^{29}$. En efecto, los pacientes con reactivación de enfermedad de Chagas e infección por VIH presentan cargas parasitarias significativamente más altas que los pacientes con ambas condiciones pero sin reactivación (medianas de 1.428 copias/mL versus 1,57 copias/ $\mathrm{mL}$; respectivamente) $)^{29}$. Así, las 1.400 copias $/ \mathrm{mL}$ de $T$. cruzi detectadas en nuestro paciente en el compartimento sanguíneo están en concordancia con estos estudios. Las mediciones cuantitativas parasitarias por RPC en sangre también han sido utilizadas en el seguimiento de pacientes tratados por enfermedad de Chagas ${ }^{30}$. En contraste, la detección parasitaria cuantitativa por RPC ha sido muy poco aplicada en muestras de $\mathrm{LCR}^{10}$. Así, la detección de una carga notoriamente elevada en el LCR en nuestro paciente respecto a los valores en sangre, sugiere su valor diagnóstico y representa una aproximación novedosa. Las pruebas serológicas en sangre tienen una utilidad indirecta en el diagnóstico ya que los cuadros de meningoencefalitis o masas cerebrales pueden tener otras causas alternativas, incluyendo toxoplasmosis, linfoma del SNC, infección por Mycobacterium tuberculosis o Cryptococcus neoformans. Además, se han descrito casos de granulomas por T. cruzi con serología negativa y cerca de $5 \%$ de los pacientes con VIH y co-infección chagásica tienen serología negativa ${ }^{17,26,31}$. 
La posibilidad de una toxoplasmosis cerebral en nuestro caso, no puede ser descartada totalmente debido a la regresión inicial de los granulomas frontales luego de iniciar una terapia específica. Sin embargo, esta posibilidad no pudo ser confirmada por RPC en muestras de sangre o LCR, aunque el largo tiempo transcurrido entre el inicio de la terapia con cotrimoxazol y su búsqueda en la segunda punción lumbar (40 días), pudo haber generado un resultado negativo. Se han descrito algunos casos similares al nuestro, con doble serología parasitaria positiva donde también se ha optado por tratar inicialmente al paciente por una presunta toxoplasmosis cerebral $^{26}$. Por otra parte, no creemos que un SIRI pueda explicar el deterioro del paciente ya que no se cumplen los criterios sugeridos para su reconocimiento ${ }^{32}$. Así, no hubo un aumentó en el recuento de los linfocitos CD4 (aunque pudiera estar limitado por uso de corticoesteroides), el paciente no tuvo una mejoría inicial, la evolución fue explicable por la historia natural de su infección presente desde comienzo y los eventos sucedieron en un lapso muy breve $^{32}$. Todos estos argumentos deben cumplirse para consignar una reconstitución inmune, lo que no ocurrió en nuestro paciente.

Benznidazol y nifurtimox son los únicas fármacos con eficacia demostrada sobre T. cruzi. Nifurtimox se asocia a efectos adversos gastrointestinales, irritabilidad, insomnio, cambios de ánimo y neuropatía periférica dosis dependiente. La dosis habitual es $8-10 \mathrm{mg} / \mathrm{Kg}$ /día por vía oral, dos a tres meses o terapias más prolongadas en pacientes con inmunosupresión persistente ${ }^{14}$. No obstante, la letalidad descrita en el compromiso chagásico del SNC por reactivación oscila entre $55-100 \%$ con una mediana de sobrevida cercana al mes ${ }^{17,25,31}$. Se han registrado algunos casos sobrevivientes ocasionales asociados a un diagnóstico y terapia antiparasitaria precoz y a la recuperación inmune luego del inicio del tratamiento antirretroviral ${ }^{14,25,33}$. No existe información sobre la cual apoyar recomendaciones de quimioprofilaxis en pacientes con serología positiva.

La única recomendación universal es iniciar la TARV ya que la recuperación inmune permite evitar la reactivación.

Al respecto existen algunas directrices ministeriales que permiten establecer una estrategia terapéutica encaminada a disminuir al máximo la carga circulante o de reserva de $T$. cruzi en un hospedero inmunocomprometido, para así impedir futuras reactivaciones y evitar que se desarrolle un SIRI al restaurar la inmunidad después de iniciar TARV.

Las guías incluyen la solicitud de serología de Chagas en el control inicial de pacientes con ELISA VIH reactivo confirmado, lo que permite detectar precozmente los pacientes co-infectados. Por otra parte, el Ministerio de Salud de Chile incluye a los pacientes inmunocomprome- tidos sin distinción de causa, con serología positiva para $T$. cruzi, en los grupos prioritarios de manejo farmacológico. Desde el año 2015 se dispone tanto de nifurtimox como benznidazol en los centros de referencia para el manejo farmacológico de la enfermedad de Chagas. Así, todo paciente con VIH/SIDA y serología para $T$. cruzi positiva confirmada, debería ser derivado a estos centros para recibir una evaluación y tratamiento farmacológico en el momento de la detección, con lo que se puede lograr mantener una baja carga parasitaria.

En pacientes con recuentos bajos de linfocitos CD4 se sugiere postergar el inicio de la TARV para minimizar el riesgo de una respuesta inmune paradójica ${ }^{14}$, incluso algunos autores recomiendan completar 60 días con nifurtimox antes de iniciar la TARV. Por el riesgo de reactivación luego de una respuesta inicial se recomienda también continuar con una profilaxis secundaria trisemanal con el mismo medicamento hasta alcanzar valores de linfocitos CD4 > 200 cél $/ \mathrm{mm}^{335}$.

Los casos de reactivación chagásica en pacientes con infección por VIH/SIDA en Chile son muy escasos y se remontan a los comienzos de la pandemia de SIDA con un curso inexorablemente fatal a pesar de una respuesta transitoria a fármacos antiparasitarios y a otro caso más reciente con terapia y sobrevida exitosa ${ }^{13-16,34}$.

En conclusión, la reactivación de $T$. cruzi es uno de los diagnósticos posibles en pacientes que se presentan con infección VIH/SIDA y una lesión de tipo tumoral o granulomatosa en el SNC. Apoyan esta posibilidad, la exposición epidemiológica, la serología positiva y la distribución de las lesiones en los estudios de imágenes. El diagnóstico puede confirmarse no sólo por métodos convencionales sino que también por técnicas moleculares cuantitativas. A pesar del mal pronóstico global, se puede intentar terapia específica previa o junto a la TARV.

Agradecimientos: Los autores desean agradecer el excelente apoyo técnico de los Srs. Daniel Escobar Araya y Egon Álvarez Vargas, profesionales del Laboratorio de Biología Molecular de la Sección Parasitología del ISP.

\section{Resumen}

La reactivación de la infección por Trypanosoma cruzi es un diagnóstico infrecuente pero posible en pacientes con infección por VIH/SIDA y una lesión de tipo tumoral o granulomatosa en el sistema nervioso central. Presentamos el caso clínico de un paciente de 60 años con VIH/ SIDA y recuentos bajos de linfocitos CD4, con síntomas cerebelosos y paresia leve, lesiones hipodensas supra e infratentoriales y serología positiva para Toxoplasma gondii y $T$. cruzi. Se trató empíricamente como una toxoplasmosis cerebral y con terapia antiretroviral, sin 
respuesta clínica. La enfermedad de Chagas cerebral se confirmó por RPC cuantitativa en el LCR. El paciente falleció a pesar de recibir terapia con nifurtimox. Apoyan la posibilidad de un Chagas cerebral en pacientes con VIH/SIDA, la exposición epidemiológica, la serología positiva y el patrón de distribución de las lesiones en las imágenes. El diagnóstico puede mejorarse con técnicas moleculares cuantitativas en LCR. A pesar de su mal pronóstico, se puede intentar una terapia específica junto al tratamiento antiretroviral.

\section{Referencias bibliográficas}

1.- WHO. Chagas diseases (American Trypanosomiasis) Fact sheet. Disponible en http://www.who.int/mediacentre/factsheets/ fs340/en/ (Fecha de acceso: 11 de enero de 2017)

2.- Martins-Melo F R, Lima Mda S, Ramos A N Jr, Alencar C H, Heukelbach J. Prevalence of Chagas disease in pregnant women and congenital transmission of Trypanosoma cruzi in Brazil: a systematic review and meta-analysis. Trop Med Int Health 2014; 19 : 943-57.

3.- Toso A, Vial F, Galanti N. Transmisión de la enfermedad de Chagas por vía oral. Rev Med Chile 2011; 139: 258-66.

4.- Gómez-P C F, Mantilla-H J C, RodriguezMorales J C. Fatal Chagas disease among solid-organ transplant recipients in Colombia. Open Forum Infect Dis 2014; 1: ofu032.

5.- Kransdorf E P, Zakowski P C, Kobashigawa J A. Chagas disease in solid organ and heart transplantation. Curr Opin Infect Dis 2014; 27 : 418-24.

6.- Angheben A, Boix L, Buonfrate D, Gobbi F, Bisoffi Z, Pupella S, et al. Chagas disease and transfusion medicine: a perspective from non-endemic countries. Blood Transfus 2015; 13: 540-50

7.- Huprikar S, Bosserman E, Patel G, Moore A, Pinney S, Anyanwu A, et al. Donor-derived Trypanosoma cruzi infection in solid organ recipients in the United States, 2001-2011.

Am J Transplant 2013; 13: 2418-25.

8.- Chagas Vigilancia Vectorial Boletín ISP. Disponible en http://www.ispch.cl/sites/default/ files/Chagas\%2023-06-2014_0.pdf (Fecha de acceso: 11 de enero de 2017)

9.- Lin M H, Chen T C, Kuo T T, Tseng C C, Tseng C P. Real-time PCR for quantitative detection of Toxoplasma gondii. J Clin Microbiol 2000; 38: 4121-5.

10.- Qvarnstrom Y, Schijman A G, Veron V, Aznar C, Steurer F, da Silva A J. Sensitive and specific detection of Trypanosoma cruzi DNA in clinical specimens using a multi-target realtime PCR approach. Plos Negl Trop Dis 2012; 6: e1689.

11.- Piron M, Fisa R, Casamitjana N, LopezChejade P, Puig L, Vergés M, et al. Development of a real-time PCR assay for
Trypanosoma cruzi detection in blood samples. Acta Trop 2007; 103:195-200.

12.- Departamento de Epidemiología, División de Palnificación Sanitaria. Ministerio de Salud de Chile. Diciembre 2013. Informe Nacional: evolución de la infección por VIH/SIDA, Chile 1984-2012. Rev Chilena Infectol 2015; 32 (Supl 1): 17-43.

13.- Oddó D, Casanova M, Acuña G, Ballesteros J, Morales B. Acute Chagas disease (Trypanosomiasis Americana) in acquired immunodeficiency syndrome: report of two cases. Human Pathology 1992; 23: 41-4.

14.- López O. Meningoencefalitis chagásica en un paciente con infección por VIH/SIDA con sobrevida a tres años: caso clínico. Rev Chilena Infectol 2010; 27:160-4.

15.- Labarca J, Acuña G, Leiva J, Cambon G, Oddó B, Sepúlveda C, et al. Enfermedad de Chagas en el síndrome de inmunodeficiencia adquirida. Casos clínicos. Rev Méd Chile 1992; 120: 174-9.

16.- Torrealba G, Acuña G, Tagle P, Tapia J, Huete I. Valor de la biopsia cerebral en pacientes con SIDA y lesiones expansivas cerebrales. Rev Méd Chile 1990; 118: 1367-71.

17.- Almeida D A, Ramos Júnior A N, Correia D, Shikani-Yasuda MA. Co-infection Trypanosoma cruzi/HIV: systematic review (1980-2010).

Rev Soc Bras Med Trop 2011; 44: 762-70.

18.- Verdugo F, Pinto F, Charpentier P, Von Mühlenbrock C, Soto A, Dabanch J, et al. Pacientes con infección por VIH/SIDA en una Unidad de Pacientes Críticos. La experiencia de un hospital general en un país en vías de desarrollo. Rev Chilena Infectol 2015; 32: 294303.

19.- Lazo J E, Meneses A C, Rocha A, Frenkel J K, Marquez J O, Chapadeiro E, et al. Toxoplasmic and chagasic meningoencephalitis in patients with human immunodeficiency virus infection: anatomopathologic and tomographic differential diagnosis. Rev Soc Bras Med Trop 1998; 31:163-71.

20.- Chimelli L. A morphological approach to the diagnosis of protozoal infections of the central nervous system. Patholog Res Int 2011; 2011: 29A853.

21.- Rocha M, Ferreira M S, Nishioka S A, Silva A M, Burgarelli M K, Silva M. et al. Trypanosoma cruzi meningoencephalitis and miocarditis in patient with acquired immunodeficiency syndrome Rev Inst Med Trop S Paulo 1993; 35: 205-8.

22.- Del Valle Pugliese Uliarte D, Trombetta Durante L A, Moreno Rivas D, Galache Villegas V, Semorile Maestre K, Bava de Soto A J. Miocarditis aguda y meningoencefalitis por Trypanosoma cruzi en un paciente seropositivo al VIH. Rev Cubana Med Trop 2014; 66: 458-64.

23.- Del Castillo M, Mendoza G, Oviedo J, Pérez Blanco R P, Anselmo A E, Silva M. AIDS and Chagas disease with central nervous system tumor-like lesion. Am J Med 1990; 88: 693-4.

24.- Pimentel P C, Handfas B W, Carmignani M. Trypanosoma cruzi meningiencephalitis in AIDS mimicking cerebral metastasis. Arq Neuropsiquiatr 1996; 54:102-6.

25.- Bern C. Chagas disease in the immunosuppressed host. Curr Opin Infect Dis 2012; 25:450-7.

26.- Sica R E, Gargiullo G, Papayanis C. Tumourlike chagasic encephalitis in AIDS patients. Arq Neuropsiquiatr 2008; 66:881-4.

27.- Catay E R, Mizdraji G R, Esteban H, Popescu B. Meningoencefalitis chagásica seudotumoral en SIDA. Medicina (B Aires) 2010; 70: 261.

28.- Madalosso G, Pellini A C, Vasconcelos M J, Ribeiro A F, Weissmann L, Oliveira Filho G S, et al. Chagasic meningoencephalitis: case report of a recently included AIDS-defining illness in Brazil. Rev Inst Med Trop Sao Paulo 2004; 46: 199-202.

29.- de Freitas V L, da Silva S C, Sartori A M, Bezerra R C, Westphalen E V, Molina T D, et al. Real-time PCR in HIV/Trypanosoma cruzi coinfection with and without Chagas disease reactivation: association with HIV viral load and CD4 level. PLoS Negl Trop Dis 2011; 5: e1277.

30.- Bianchi F, Cucunubá Z, Guhl F, González N L, Freilij H, Nicholls R S, et al. Follow-up of an asymptomatic Chagas disease population of children after treatment with nifurtimox (Lampit) in a sylvatic endemic transmission area of Colombia. PLoS Negl Trop Dis 2015; 9: e0003465.

31.- Lattes R, Lasala MB. Chagas disease in the immunosuppressed patient. Clin Microbiol Infect 2014; 20: 300-9.

32.- Haddow L J, Easterbrook P J, Mosam A, 
Khanyile N G, Parboosing R, Moodley P, et al. Defining immune reconstitution inflammatory syndrome: evaluation of expert opinion versus 2 case definitions in a South African Cohort. Clin Infect Dis 2009; 49: 1424-32.

33.- Corti M, Yampolsky C. Prolonged survival and immune reconstitution after chagasic meningoencephalitis in a patient with acquired immunodeficiency syndrome. Rev Soc Bras Med Trop 2006; 39: 85-8.

34.- Solari A, Saavedra H, Sepúlveda C, Oddó D, Acuña G, Labarca J, et al. Successful treatment of Trypanosoma cruzi encephalitis in a patient with hemophilia and AIDS. Clin Infect Dis 1993; 16: 255-9.

35.- Cecchini D M, Lespada M I, Riarte A,
Rodríguez C G. Reactivación de la enfermedad de Chagas en el sistema nervioso central de pacientes infectados con el virus de la inmunodeficiencia humana. Actualizaciones en SIDA. Buenos Aires. 2009; Vol 17 (64): 52-9. Disponible en https://www.huesped.org.ar/wpcontent/uploads/2014/09/ASEI-64-52-59.pdf (Fecha de acceso: 11 de enero de 2017). 